\title{
Strain and temperature dependencies of multimodal interference spectra in hetero-core-fiber structures
}

\author{
Yosuke Mizuno ${ }^{1,2^{*}}$, Sonoko Hagiwara ${ }^{2}$, Heeyoung Lee $^{3}$, Neisei Hayashi ${ }^{2}$, Michiko \\ Nishiyama ${ }^{4}$, Kazuhiro Wanatabe ${ }^{4}$, and Kentaro Nakamura ${ }^{2}$ \\ 1 Faculty of Engineering, Yokohama National University, Yokohama 240-8501, Japan \\ 2 Institute of Innovative Research, Tokyo Institute of Technology, Yokohama 226-8503, Japan \\ 3 College of Engineering, Shibaura Institute of Technology, Tokyo 135-8548, Japan \\ 4 Faculty of Science and Engineering, Soka University, Tokyo 192-8577, Japan \\ *E-mail: mizuno-yosuke-rg@ynu.ac.jp
}

\begin{abstract}
We investigate, for the first time, the temperature and strain dependencies of the spectral dips of the multimodal interference patterns in hetero-core-fiber structures at telecom wavelength. With increasing temperature and strain, the spectral dips shifted to shorter wavelengths with dependence coefficients of $-3.5 \mathrm{~nm} /{ }^{\circ} \mathrm{C}$ and $-0.62 \mathrm{pm} / \mu \varepsilon$, respectively. Their absolute values were $\sim 60$ times larger and $\sim 30$ times smaller than the temperature and strain sensitivities of a standard single-mode-multimode-single-mode sensor, respectively. This result implies that the hetero-core-fiber structures can be potentially used to develop highly sensitive temperature sensors with reduced strain sensitivity.
\end{abstract}

Among a variety of optical fiber sensors that have been developed so far for structural health monitoring, ${ }^{1-6)}$ we focus on what we call a hetero-core fiber structure, in which a small-core single-mode fiber (SMF) is sandwiched (spliced) between two normal-core SMFs. ${ }^{7-11)}$ By exploiting the optical loss caused by bending, the hetero-core structure has been used as a power-based bending-angle sensor. It has a lot of advantages, such as insusceptibility to temperature, ${ }^{7)}$ high-speed operation, ${ }^{8)}$ and controllability of sensitivity by the core diameter, etc., ${ }^{9)}$ and has been used to develop numerous commercially available devices. The hetero-core fiber sensors will be more useful in practical applications, if temperature or strain can be measured in addition to bending angle while the sensor structure is maintained.

One method for achieving this aim is to exploit the multimodal interference in the hetero-core-fiber structure - a phenomenon in which multiple propagating lights with 
different modes in multimode fibers (MMFs) interfere with one another. ${ }^{12)}$ Consequently, the spectrum of the transmitted light exhibits characteristic peaks and dips, which shift by temperature change or applied strain. Therefore, by measuring the shift of one (or some) of the peaks/dips, temperature and strain can be estimated. One of the simplest configurations for implementing modal-interference-based sensors is a so-called single-mode-multimode-single-mode (SMS) structure. ${ }^{13-20)}$ This is in analogy with the hetero-core-fiber structure, in which multiple propagation modes exist in the cladding layer of the small-core SMF in addition to the core mode. Although the multimodal interference in the hetero-core-fiber structures has been already observed ${ }^{21)}$ and its dependencies on refractive index and humidity have been clarified, ${ }^{22,23)}$ its strain and temperature sensing characteristics remain to be unclarified.

In this work, we investigate the temperature and strain dependencies of the interference spectra in the hetero-core-fiber structures. We find that, at $\sim 1580 \mathrm{~nm}$, with increasing temperature and strain, a characteristic dip shifts to shorter wavelengths with dependence coefficients of $-3.5 \mathrm{~nm} /{ }^{\circ} \mathrm{C}$ and $-0.62 \mathrm{pm} / \mu \varepsilon$, respectively. These values indicate that, compared to conventional silica-fiber-based SMS sensors, hetero-core-fiber-based SMS sensors may enable highly sensitive temperature measurement with small strain sensitivity.

The operating principle of SMS-based temperature and strain sensing is simple. When light is injected from the first SMF into an MMF (sensing head), multiple modes are excited and travel along the MMF with their own propagation constants. At the other end of the MMF, the net field coupled to the second SMF is determined by the relative phase differences among all the propagation modes. Under the assumption that the MMF and the two SMFs are perfectly axially aligned, the optical output power from the second SMF, $P_{\text {out }}$, can be given as ${ }^{18)}$

$$
P_{\text {out }}=\left|a_{0}^{2}+a_{1}^{2} \exp i\left(\beta_{0}-\beta_{1}\right) L+a_{2}^{2} \exp i\left(\beta_{0}-\beta_{2}\right) L+\cdots\right|^{2},
$$

where $a_{j}$ is the field amplitudes of the $j$-th modes at the first SMF-to-MMF boundary, $\beta_{j}$ is the propagation constants of the $j$-th modes, and $L$ is the length of the MMF. It is clear that $P_{\text {out }}$ is influenced by temperature change and strain applied to the MMF (with some change in $\beta_{j}$ and $L$ ). Thus, temperature and strain sensing can be performed by measuring the shift of the spectral dips (or peaks) of the modal interference pattern. Considering that multiple propagation modes exist in the small-core fiber of the hetero-core-fiber structure, temperature and strain sensing should be feasible in the same manner.

The experimental setup is depicted in Fig. 1. We used the hetero-core-fiber structures involving small-core SMFs (core diameter: $3 \mu \mathrm{m}$, loss: $50 \mathrm{~dB} / \mathrm{m}$ at $488 \mathrm{~nm}$ ) spliced with 
normal-core SMFs (core diameter: $9 \mu \mathrm{m}$, loss: $0.25 \mathrm{~dB} / \mathrm{km}$ at $1550 \mathrm{~nm}$ ). The lengths of the small-core SMFs were $210 \mathrm{~mm}$ and $180 \mathrm{~mm}$ for temperature and strain characterizations, respectively. The lengths of all the normal-core SMFs were $\sim 2 \mathrm{~m}$. The $100-\mathrm{mW}$ output of a supercontinuum source (SCS; bandwidth: 460-2000 nm) was injected in the hetero-core-fiber structure, and the spectrum of the transmitted light was observed using an optical spectrum analyzer (OSA). Temperature change and strain were applied to the whole length of the MMF using a thermostatic chamber and translation stages, respectively. The room temperature was $28^{\circ} \mathrm{C}$.

We first measured and compared the optical spectra before and after transmission through the hetero-core-fiber structure (involving a 210-mm-long small-core MMF), which correspond to the SCS output and the OSA input, respectively (Fig. 2(a)). The optical power was reduced at all the wavelengths, and the amount of the power loss was much higher at longer wavelengths. This is natural considering the wavelength dependence of the propagation loss in the small-core SMF. The spectrum after transmission exhibited multiple characteristic dips that were not observed in the SCS output, especially at longer wavelengths. This is also natural considering that, at shorter wavelengths, most of the optical power is not distributed to cladding modes in the small-core SMF and that modal interference is less striking. The magnified view around $1590 \mathrm{~nm}$ is shown in Fig. 2(b). Multiple clear dips were observed in this range, and we selected the dip around $1600 \mathrm{~nm}$ in the following temperature characterization.

Then, we measured the temperature dependence of this spectral dip as shown in Fig. 3(a). The vertical axis was normalized so that the dip power became 0. With increasing temperature, the spectral dip shifted to shorter wavelengths. The dip wavelength dependence on temperature shown in Fig. 3(b) was almost linear with a coefficient of $-3.5 \mathrm{~nm} /{ }^{\circ} \mathrm{C}$. The linear coefficient of determination $\left(r^{2}\right.$, the square of the correlation coefficient) was as high as 0.999. We also measured the strain dependence of the spectral dip around $1579 \mathrm{~nm}$ (note that one of the clear dips was observed at this wavelength when another sample was used) as shown in Fig. 4(a). As strain increased, the dip also shifted to shorter wavelengths. The dip wavelength dependence on strain (Fig. 4(b)) shows that the strain coefficient was -0.62 $\mathrm{pm} / \mu \varepsilon$; the $r^{2}$ value was 0.998 .

Subsequently, in addition to the measurement around $1579 \mathrm{~nm}$, we measured the strain dependencies of the spectra and the dip wavelengths around $1486 \mathrm{~nm}, 1517 \mathrm{~nm}$, and 1549 nm (Figs. 5(a)-(f)). The calculated strain coefficients (the $r^{2}$ values were all higher than 0.95) were then plotted as a function of the dip wavelength at zero strain (Fig. 6). It is clear 
that the strain sensitivity does not largely depend on the wavelength in this range.

Finally, to consider the sensing performance, let us compare the absolute values of the obtained sensitivities with those of a standard silica-based SMS sensor. ${ }^{13)}$ The obtained temperature coefficient $\left(3.5 \mathrm{~nm} /{ }^{\circ} \mathrm{C}\right)$ is approximately 60 times larger than the previously reported value $\left(59 \mathrm{pm} /{ }^{\circ} \mathrm{C}\right)$, whereas the obtained strain coefficient $(0.62 \mathrm{pm} / \mu \varepsilon)$ is approximately 30 times smaller than that previously reported $(19 \mathrm{pm} / \mu \varepsilon)$. Note that, although the previously reported values were obtained at different MMF lengths and wavelengths, these two factors have been reported not to have clear influence on the sensitivities. ${ }^{17,19)}$ This result indicates a potential of highly sensitive temperature sensing with reduced strain sensitivity.

In conclusion, we investigated the temperature and strain dependencies of the spectral dips of the multimodal interference patterns in the hetero-core-fiber structures. At $\sim 1580 \mathrm{~nm}$, as temperature change and strain increased, the spectral dips shifted to shorter wavelengths with dependence coefficients of $-3.5 \mathrm{~nm} /{ }^{\circ} \mathrm{C}$ and $-0.62 \mathrm{pm} / \mu \varepsilon$, respectively. Their absolute values were $\sim 60$ times larger and $\sim 30$ times smaller than the temperature and strain sensitivities of a standard SMS sensor previously reported, respectively; this result indicates that the multimodal interference in the hetero-core-fiber structures may be used to develop highly sensitive temperature sensors with small strain sensitivity. Considering that the hetero-core-fiber sensors have been used to measure bending angle based on power information, this result that temperature can be measured with high sensitivity based on frequency information indicates that this structure could be exploited for simultaneous sensing of temperature and bending angle. Combined use of multimodal interference and Fabry-Perot interference will be another interesting topic to be discussed in future.

\section{Acknowledgments}

This work was supported by JSPS KAKENHI Grant Numbers $17 \mathrm{H} 04930$ and $17 \mathrm{~J} 07226$. 


\section{References}

1) G. Rajan (Ed.), Optical Fiber Sensors: Advanced Techniques and Applications (CRC Press, Boca Raton, 2015).

2) A. H. Hartog, An Introduction to Distributed Optical Fibre Sensors (CRC Press, Boca Raton, 2017).

3) H. Alemohammad, Opto-Mechanical Fiber Optic Sensors: Research, Technology, and Applications in Mechanical Sensing (Elsevier, Oxford, 2018).

4) R. Kashyap, Fiber Bragg Gratings (Elsevier, San Diego, 1999).

5) P. F. C. Antunes, C. A. Marques, H. Varum, and P. S. Andre, IEEE Sens. J. 12, 2399 (2012).

6) A. G. Leal-Junior, A. Frizera, C. Marques, and M. J. Pontes, IEEE Sens. J. 20, 569 (2020).

7) H. Sasaki, Y. Kubota, and K. Watanabe, SICE Trans. 40, 981 (1997).

8) M. Nishiyama and K. Watanabe, Sens. Actuators, A Phys. 209, 154 (2014).

9) H. Sasaki, Y. Kubota, and K. Watanabe, SICE Trans. Ind. Appl. 4, 25 (2005).

10) H. Yamazaki, M. Nishiyama, K. Watanabe, and M. Sokolov, Sens. Actuators, A Phys. 247, 98 (2016).

11) M. Nishiyama, M. Sonobe, and K. Watanabe, Biomed. Opt. Express 7, 3675 (2016).

12) O. Frazão, S. O. Silva, J. Viegas, L. A. Ferreira, F. M. Araújo, and J. L. Santos, Appl. Opt. 50, E184 (2011).

13) Y. Liu and L. Wei, Appl. Opt. 46, 2516 (2007).

14) S. M. Tripathi, A. Kumar, R. K. Varshney, Y. B. P. Kumar, E. Marin, and J. P. Meunier, J. Lightwave Technol. 27, 2348 (2009).

15) J. Huang, X. Lan, H. Wang, L. Yuan, T. Wei, Z. Gao, and H. Xiao, Opt. Lett. 37, 4308 (2012).

16) G. Numata, N. Hayashi, M. Tabaru, Y. Mizuno, and K. Nakamura, IEEE Photon. J. 6, 6802306 (2014).

17) G. Numata, N. Hayashi, M. Tabaru, Y. Mizuno, and K. Nakamura, Appl. Phys. Express 8, 072502 (2015).

18) A. Kumar, R. K. Varshney, C. S. Antony, and P. Sharma, Opt. Commun. 219, 215 (2003).

19) T. Kawa, G. Numata, H. Lee, N. Hayashi, Y. Mizuno, and K. Nakamura, IEICE Electron. Express 14, 20161239 (2017).

20) T. Kawa, G. Numata, H. Lee, N. Hayashi, Y. Mizuno, and K. Nakamura, Jpn. J. Appl. Phys. 56, 078002 (2017).

21) O. V. Ivanov, Opt. Commun. 282, 3895 (2009).

22) Q. Wu, Y. Semenova, P. Wang, and G. Farrell, J. Opt. 13, 125401 (2011).

23) Q. Wu, Y. Semenova, J. Mathew, P. Wang and G. Farrell, Opt. Lett. 36, 1752 (2011). 


\section{Figure Captions}

Fig. 1. (Color online) Experimental setup for observing multimodal interference caused in hetero-core-fiber structure. OSA: optical spectrum analyzer, SCS: supercontinuum source, SMF: single-mode fiber.

Fig. 2. (Color online) (a) Optical spectra measured before and after transmission through the hetero-core-fiber structure. (b) Its magnified view around $1590 \mathrm{~nm}$. The spectral dip in the red circle was used in the temperature characterization.

Fig. 3. (Color online) (a) Temperature dependence of the spectral dip (normalized). (b) Dip wavelength plotted as a function of temperature. The solid line is a linear fit.

Fig. 4. (Color online) (a) Strain dependence of the spectral dip. (b) Dip wavelength plotted as a function of strain. The solid line is a linear fit.

Fig. 5. (Color online) Strain dependencies of the spectral dips around (a) $1486 \mathrm{~nm}$, (b) $1517 \mathrm{~nm}$, and (c) $1549 \mathrm{~nm}$. Dip wavelength dependencies on strain around (d) $1486 \mathrm{~nm}$, (e) $1517 \mathrm{~nm}$, and (f) $1549 \mathrm{~nm}$. The solid lines are linear fits.

Fig. 6. (Color online) Strain sensitivity plotted as a function of the dip wavelength at zero strain. 


\section{Figures}

Fig. 1.

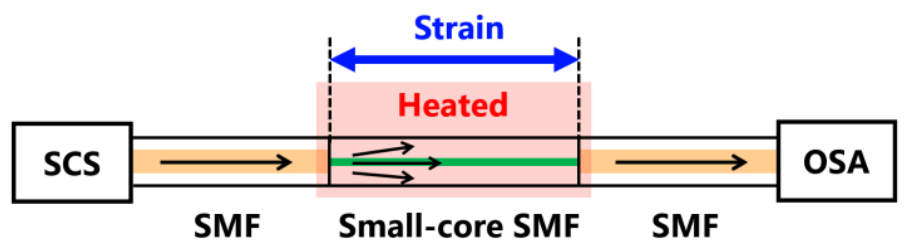

Fig. 2.
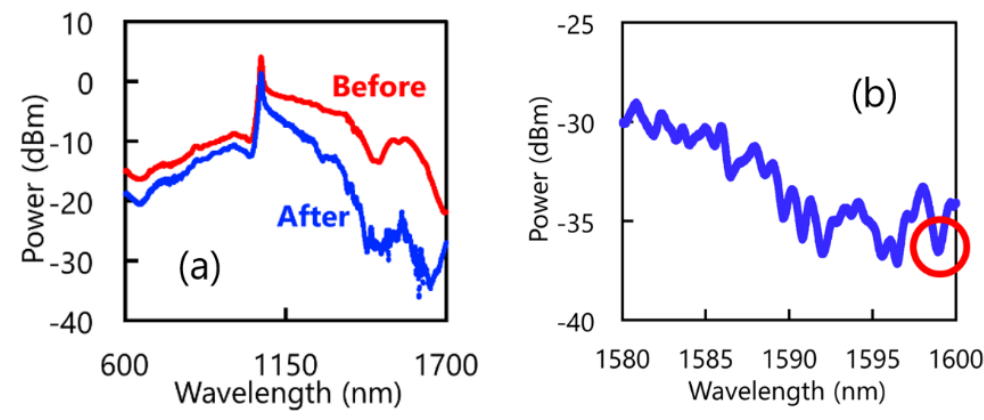

Fig. 3
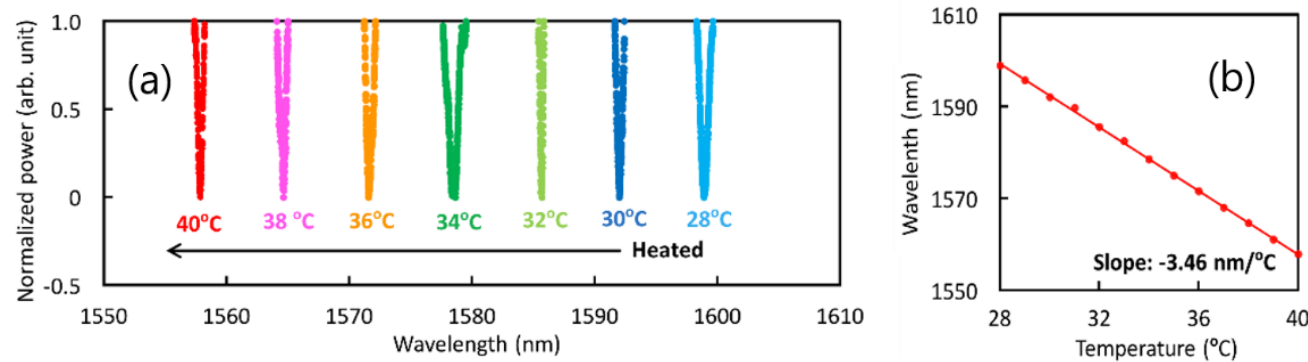

Fig. 4
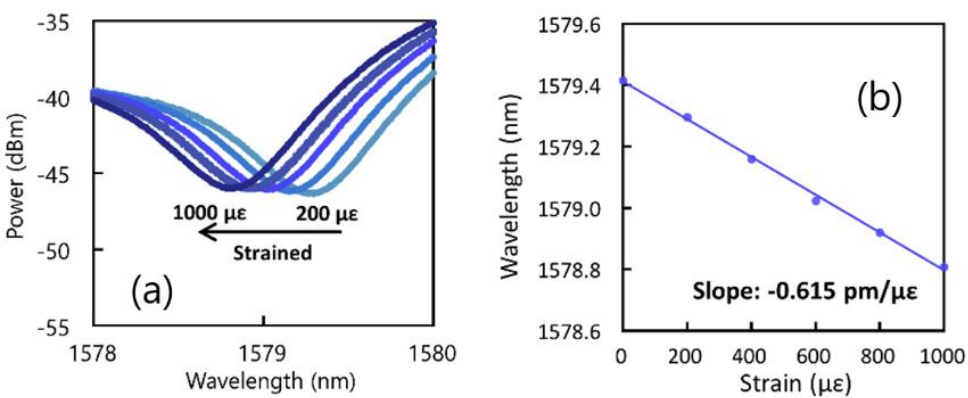
Fig. 5
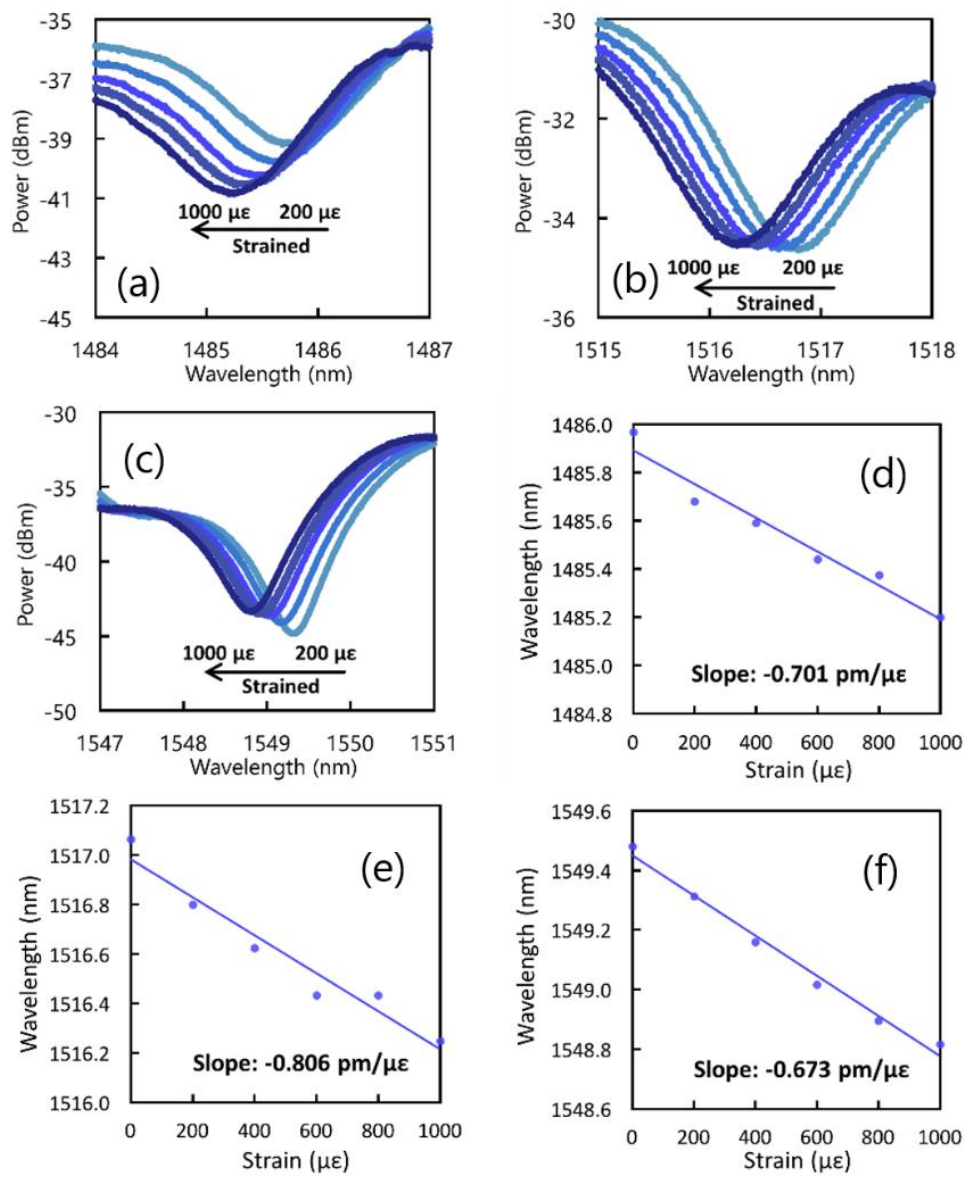

Fig. 6

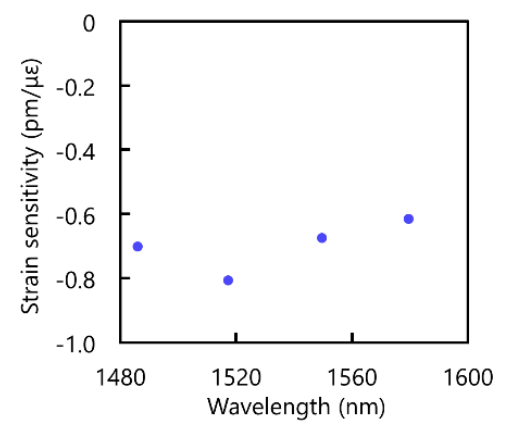

